\title{
Ectodysplasin A/Ectodysplasin A Receptor System and Their Roles in Multiple Diseases
}

\author{
Zhensheng Cait, Xia Dengt, Jue Jia, Dong Wang* and Guoyue Yuan* \\ Department of Endocrinology, Affiliated Hospital of Jiangsu University, Zhenjiang, China
}

Ectodysplasin A (EDA) is a member of the tumor necrosis factor (TNF) family of ligands that was initially reported to induce the formation of various ectodermal derivatives during normal prenatal development. EDA exerts its biological activity as two splice variants, namely, EDA-A1 and EDA-A2. The former binds to the EDA receptor

OPEN ACCESS

Edited by:

Ana Cláudia Garcia De Oliveira

Duarte,

Federal University of São Carlos,

Brazi

Reviewed by:

Junichi Iwata,

University of Texas Health Science

Center at Houston, United States

Johannes W. Von den Hoff,

Radboud University Nijmegen Medical

Centre, Netherlands

*Correspondence:

Dong Wang

zjdongdonghao@aliyun.com

Guoyue Yuan

yuanguoyue@ujs.edu.cn

${ }^{\dagger}$ These authors have contributed equally to this work

Specialty section:

This article was submitted to Metabolic Physiology,

a section of the journal

Frontiers in Physiology

Received: 02 October 2021 Accepted: 18 November 2021 Published: 06 December 2021

Citation:

Cai Z, Deng X, Jia J, Wang $D$ and Yuan $G$ (2021) Ectodysplasin AVEctodysplasin A Receptor System and Their Roles in Multiple Diseases. Front. Physiol. 12:788411. doi: 10.3389/fphys.2021.788411
(EDAR), resulting in the recruitment of the intracellular EDAR-associated death domain (EDARADD) adapter protein and the activation of the NF-kB signaling pathway, while the latter binds to a different receptor, EDA2R, also known as X-linked ectodermal dysplasia receptor (XEDAR). Inactivation mutation of the EDA gene or the genes coding for its receptors can result in hypohidrosis ectodermal dysplasia (HED), a condition that is characterized by oligotrichosis, edentulosis or oligodontia, and oligohidrosis or anhidrosis. Recently, as a new liver factor, EDA is gradually known and endowed with some new functions. EDA levels were observed to be upregulated in several metabolic diseases, such as non-alcoholic fatty liver disease (NAFLD), obesity, and insulin resistance. In addition, EDA and its receptors have been implicated in tumor pathogenesis through the regulation of tumor cell proliferation, apoptosis, differentiation, and migration. Here, we first review the role of EDA and its two-receptor system in various signaling pathways and then discuss the physiological and pathological roles of EDA and its receptors.

Keywords: ectodysplasin A, ectodysplasin A receptor, signaling pathways, metabolism, skeletal muscle homeostasis, tumorigenesis

\section{INTRODUCTION}

The ectodysplasin A (EDA) gene is a member of the tumor necrosis factor (TNF) family located on the long arm of the $\mathrm{X}$ chromosome. The EDA transcript encodes the EDA protein that generates several splice variants, two of which-EDA-A1 (391 amino acids) and EDA-A2 (389 amino acids) which contain a TNF homology domain (Kere et al., 1996; Park et al., 2019). EDA-A1 is a homotrimer type II transmembrane protein consisting of a transmembrane domain, a furan protease recognition site, and a 19-repeat Gly-X-Y collagen domain (Liu et al., 2019). EDA-A1 binds to the ectodysplasin A receptor (EDAR), which contains 14 cysteine residues, of which only the 6 closest to the $\mathrm{N}$-terminus approximate the canonical TNF receptor consensus (Headon and Overbeek, 1999). EDA-A1/EDAR binding results in the recruitment of the intracellular EDARassociated death domain (EDARADD) adaptor protein (Kumar et al., 2001) and the activation of the NF- $\mathrm{BB}$ signaling pathway (Sadia, Foo et al., 2019). Mutations in any of EDA, EDAR, and 
EDARADD can contribute to hypohidrotic ectodermal dysplasia (HED), which affects 1 in 10,000-100,000 newborns (Wohlfart et al., 2016; Feng et al., 2018; Liu et al., 2019). EDA-A2, which contains 2 amino acids less than EDA-A1, binds to a distinct receptor, EDA2R [also known as X-linked ectodermal dysplasia receptor (XEDAR)], indicating that the insertion of two amino acids into the ligand is a determinant of the specificity of receptor binding (Yan et al., 2000). Like EDA-A1, EDA-A2 also activates the NF- $\kappa$ B signaling pathway. TRAF6 can be recruited to the EDA-A2/EDA2R complex and thereby participate in the activation of the IКB kinase (IKK) complex, which is necessary for the translocation of NF- $\kappa \mathrm{B}$ transcription factors into the nucleus (Yan et al., 2000). Studies have demonstrated that EDAA2 is expressed in aging adipose, artery, heart, lung, muscle, and skin tissues and is associated with apoptosis (Yang et al., 2015; de Vries et al., 2017).

Although EDA and its receptors are known to be essential for ectodermal morphogenesis, their functions in disease pathology and related pathways are not well understood. EDA was recently identified as a liver-secreted protein involved in the occurrence and development of metabolic dysfunction. Loss- and gain-offunction studies have indicated that EDA, particularly the EDAA2 isoform, regulates systemic glucose metabolism in type 2 diabetes mellitus (T2DM) (Awazawa et al., 2017). Additionally, the serum EDA-A2 level is dependent on T2DM, body mass index (BMI), and obesity (Yang et al., 2019). In patients with nonalcoholic fatty liver (NAFL) and non-alcoholic steatohepatitis (NASH), the plasma EDA content is high and is associated with deteriorating steatosis and fibrosis (Bayliss et al., 2021). In addition to the effects on HED and metabolic disorders, several studies have reported that EDA and its receptors are involved in cancer pathogenesis by regulating the apoptosis, proliferation, differentiation, and migration of cancer cells (Soraas and Stebbing, 2018; Vial et al., 2019; Li et al., 2020; Wang et al., 2020); however, this possibility remains controversial. Here, we review the role of EDA and its two-receptor system in various signaling pathways and then discuss the physiological and pathological roles associated with EDA and its receptors.

\section{MULTIPLE SIGNALING PATHWAYS ACTIVATED BY ECTODYSPLASIN A}

Recent studies have demonstrated that EDA and its receptors participate in multiple signaling pathways, including the Wnt/ $\beta$-catenin (Wang et al., 2020), c-Jun N-terminal kinase (JNK) (Sinha et al., 2002), bone morphogenetic protein (BMP)/Smad (Han et al., 2018), and fibroblast growth factor (FGF) signaling pathways (Häärä et al., 2012; Huh et al., 2013).

Wang et al. (2020) reported that EDAR promoted tumor cell proliferation by inducing $\mathrm{Wnt} / \beta$-catenin signaling. The expression levels of genes related to the $\mathrm{Wnt} / \beta$-catenin signaling pathway were upregulated in $\mathrm{EDA}^{\text {high }}$ samples. Additionally, the loss of EDAR could interfere with $\beta$-catenin signaling/luciferase activity, while silencing EDAR in colorectal cancer (CRC) cells led to a decrease in $\beta$-catenin abundance compared with that in vector shRNA-treated cells. Combined, these observations support that EDAR can activate the Wnt/ $\beta$-catenin signaling pathway. Similarly, Zhang et al. (2009) found that Wnt $/ \beta$-catenin signaling was necessary for the activation of the EDA/EDAR/NF- $\kappa$ B signaling pathway in epithelial cells, as well as the subsequent morphological and molecular events required for hair follicle development.

The EDA/EDAR/EDARADD pathway-mediated regulation of target genes is known to be dependent on the activation of the NF-kB pathway (Sadier et al., 2014); however, Sinha et al. (2002) reported that EDA2R can also activate the NF- $\mathrm{B}$ and JNK pathways in an EDA-A2-dependent manner. Transient transfection of cDNA encoding FLAG-labeled XEDAR-L or XEDAR-s subtypes resulted in similar activation of the NF- $\kappa$ B pathway, while significant activation of NF- $\kappa \mathrm{B}$ signaling was also observed in EDA-A2-treated 293F-XEDAR cells (Sinha et al., 2002). A different study demonstrated that EDA-A2 may activate NF-кB pathway by TRAF6. TRAF6 may be recruited to ligated XEDAR and contribute to activation of the IKK complex for translocation of NF- $\kappa \mathrm{B}$ transcription factors into the nucleus (Yan et al., 2000). In addition to NF-кB activation, EDA-A2/EDA2R can also activate the JNK pathway by stimulating a rapid and marked increase in JNK1 and JNK2 phosphorylation, thereby promoting the phosphorylation-induced activation of the c-Jun transcription factor.

BMP2, BMP4, and BMP7 are expressed in early dental epithelial cells and are key regulators of tooth morphogenesis (Zouvelou et al., 2009; Feng et al., 2011; Jia et al., 2016). A recent study showed that in dental epithelial cells, EDAA1 significantly induced Nkx2-3 expression in the pharyngeal floor as well as in oral cavity and branchial arch ectoderm (Biben et al., 2002). Han et al. (2018) found that BMP signaling is involved in tooth tip formation and tooth germ development by regulating cell differentiation and proliferation in the enamel node. Furthermore, Nkx2-3 transfection inhibited cell proliferation and induced the expression of Bmp2 and Bmpr2 mRNA and the phosphorylation of Smad1/5/8 in dental epithelial stem cells ( $\mathrm{M} 3 \mathrm{H} 1$ cells). These results indicated that $\mathrm{Nkx} 2-3$ was induced by EDA-A1 as a target molecule of the EDA-A1/EDAR pathway in dental epithelial cells and subsequently regulated cell proliferation through the BMP signaling pathway. The effects of BMP on EDAR have also been investigated. Mou et al. (2006) identified an EDAR/BMP activation-inhibition mechanism in which EDA-A1 upregulates EDAR expression, which subsequently induces BMPs expression, leading, in turn, to the suppression of EDAR expression. EDAR inhibited BMP rapidly and correlated with the level of phospho-Smad1/5/8, which is the activated form of intracellular transducers of BMP signals.

Microarray analysis showed that FGF20 is one of the earliest EDA-induced genes in hair placodes and, thus, is a putative transcriptional target of EDA (Fliniaux et al., 2008; Lefebvre et al., 2012). Häärä et al. (2012) demonstrated that FGF20 removal in EDA overexpression mice resulted in the appearance of a third molar, similar to the phenotype observed with EDA loss-offunction, suggesting that FGF20 has a critical role in modulating the initiation and size of posterior molars. The authors further found that EDA could rapidly induce FGF20 expression and 
FGF20 expression levels were related to EDA activity in vivo. Fgf20-null (Fgf20 $\beta \mathrm{Gal} / \beta \mathrm{Gal}$ ) and $\mathrm{Eda}^{-/-}$mice share similar molar phenotypes, indicating that FGF20 might be a key mediator and a direct target of EDA signaling transduction. Huh et al. (2013) revealed that, compared with untreated controls, the FGF20 message was increased by 3.3- and 16-fold after 2 and $4 \mathrm{~h}$ of EDA treatment, respectively. The activation of EDA in the epidermis led to an increase in FGF20 ${ }^{\beta \mathrm{Gal}}$ activity, whereas the opposite was observed with the loss of EDA/EDAR signaling. Further analysis also indicated that the absence of FGF20 led to the suppression of EDAR expression and, consequently, also EDAR signaling (Figure 1).

\section{THE ROLE OF ECTODYSPLASIN A IN HAIR DEVELOPMENT}

EDA is essential for the formation of skin appendages such as hair, teeth, sweat glands, and eyelids (Pinheiro and FreireMaia, 1994). Recent studies have demonstrated that alopecia is independently associated with many concurrent conditions such as obesity, insulin resistance, and metabolic syndrome (Marks et al., 2019). HFD treatment can lead to hair loss in both young and aging mice, and multiple inductions of the hair cycle result in more severe and irreversible hair loss. Furthermore, HFD can accelerate both the anagen and telogen phases, shorten the hair growth cycle, and induce lipid droplet accumulation, ROS generation, and NF- $\mathrm{B}$ signaling pathway activation in hair follicle stem cells (Morinaga et al., 2021). Liu et al. (2018) revealed that EDA mRNA and protein expression was higher in ear skin than in back skin. Moreover, alpaca ear hair is thick and straight with a long growth cycle, but its back hair is long, curved, and has a short growth cycle (Liu et al., 2011); these differences in growth cycle duration between the alpaca ear and back hair may be related to differential EDA expression. Kwack et al. (2019) explored the role of EDA-A2 and its receptor in hair follicles of mice and cultured human hair follicles. Compared with controls, the expression of the anti-apoptotic protein Bcl2 was found to be decreased after rhEDA-A2 treatment, whereas that of the pro-apoptotic Bax and cleaved caspase-3 proteins was

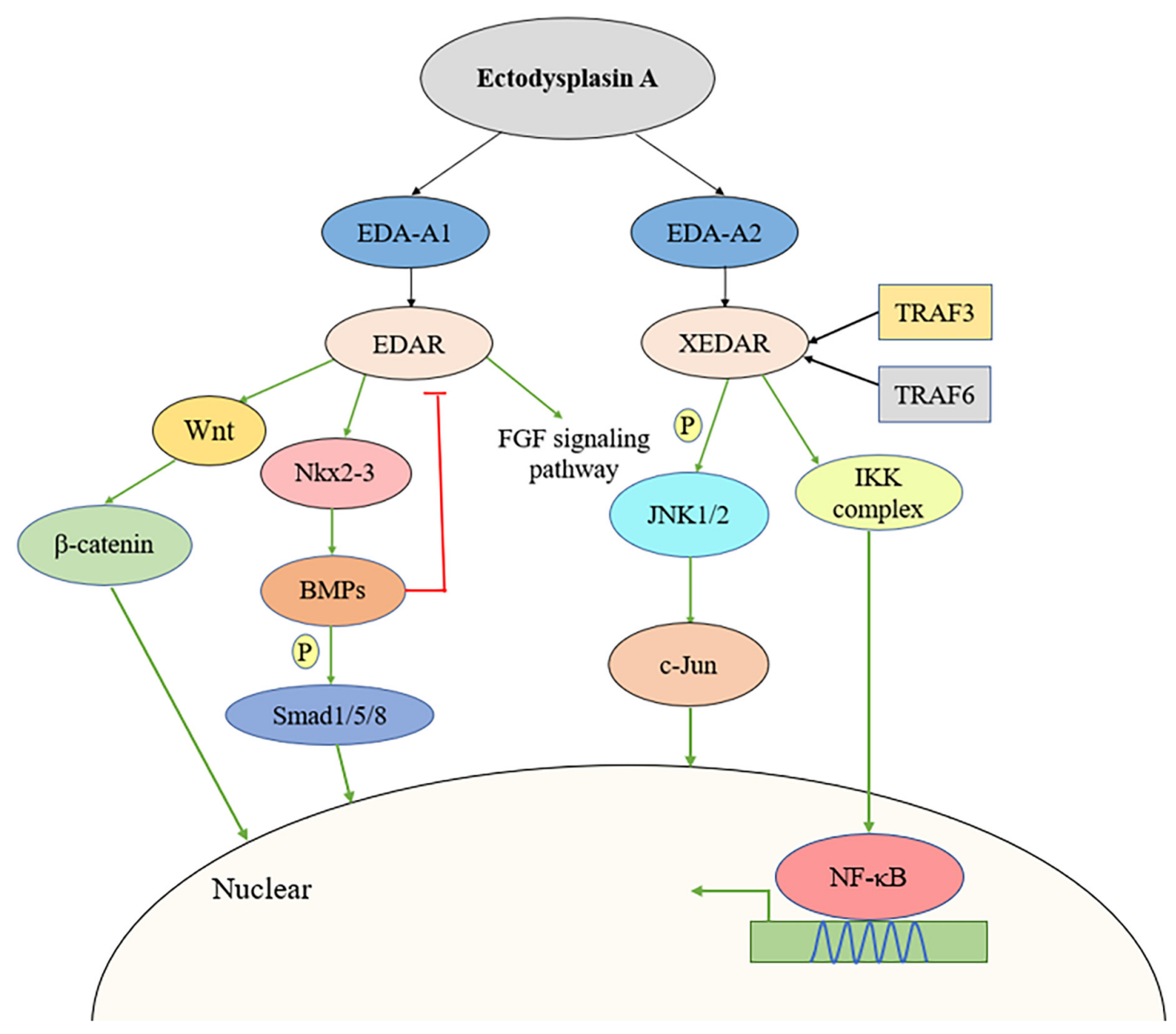

FIGURE 1 | Multiple signaling pathways activated by EDA including Wnt/B-catenin signaling pathway, JNK pathway, BMP/Smad pathway, and FGF pathway. BMP, bone morphogenetic protein; FGF, fibroblast growth factor; JNK, c-Jun N-terminal kinase. 
significantly increased. EDA2R mRNA expression peaked in the late anagen phase. Consistent with this, skin hair follicles injected with EDA-A2 showed catagen, compared with the anagen in skin injected with heat-inactivated EDA-A2. Similarly, Jiang et al. (2012) demonstrated that EDA mRNA expression in hair follicles of goat skin tissue was higher during the catagen stage than during the telogen and anagen phases. These results indicate that EDA can induce the apoptosis of hair follicle cells and regulate the hair follicle growth cycle. However, the relationship among obesity, EDA, and alopecia is not clear. Whether a HFD can lead to alopecia by affecting the expression of EDA need to be further explored, as do the associated regulatory mechanisms.

\section{THE ROLE OF ECTODYSPLASIN A IN SKELETAL MUSCLE HOMEOSTASIS}

Skeletal muscle is extremely important in regulating whole-body energy expenditure and determining resting energy expenditure. It is the main site for glucose metabolism, fatty acid oxidation, and insulin activity and an organ with high adaptability to environmental pressures, such as obesity (Zurlo et al., 1990; Stewart and Rittweger, 2006). The skeletal muscle is heterogeneous and consists of different fiber phenotypes of varying oxidative and glycolytic properties (Simcocks et al., 2020). Targeting skeletal muscle is a possible therapeutic strategy for improving metabolic homeostasis (Rossi et al., 2016). Newton et al. (2004) found that EDA-A2 might play a role in skeletal muscle homeostasis in an EDA2R expressiondependent manner. Myosin light-chain 2 (MLCH). EDA-A2 transgenic mice showed skeletal muscle degeneration in both weight-bearing and non-weight-bearing muscles. Importantly, EDA2R deficiency alleviated myodegeneration due to EDA-A2 overexpression. Additionally, a comparative analysis of EDA2R expression between skeletal muscle from wild-type and MLC2. EDA-A2 transgenic mice using an EDA2R riboprobe revealed that the EDA2R signal was strongest at sites of muscle damage in EDA-A2 transgenic mice. Recombinant human EDA-A2 can promote I $\mathrm{B} \alpha$ phosphorylation in normal human skeletal muscle cells, suggesting that EDA-A2 might cause muscle degeneration through I $\mathrm{B} \alpha$ phosphorylation; however, how EDA-A2 might exert these myodegenerative effects remains unknown. Studies have also demonstrated that treating $293 \mathrm{E}$ cells with recombinant human EDA-A2 can result in the activation of the IKK complex, leading to the phosphorylation of Iк $\mathrm{B} \alpha$ (Yan et al., 2000). In conclusion, EDA-A2 might instigate skeletal muscle degeneration by promoting $\mathrm{I} \kappa \mathrm{B} \alpha$ phosphorylation through EDA2R. I $\mathrm{B} \alpha$ might be the downstream mechanism of EDA-A2, but whether it is involved in other diseases and deeper mechanisms need to be further explored.

\section{THE ROLE OF ECTODYSPLASIN A IN METABOLIC DISEASES}

Glucose and lipid metabolism are closely linked and the stability of these two metabolic pathways is critical for maintaining body organ function ( $\mathrm{Du}$ et al., 2021). Insulin resistance is highly associated with the occurrence and development of glucose metabolism disorder (James et al., 2021). Insulin resistance is defined as a reduction in the metabolic response of insulin-responsive cells to insulin or an impaired/reduced response of blood glucose levels to circulating insulin at the systemic level (Czech, 2017). In the liver, insulin not only regulates glucose production and utilization, but also has a broader influence on lipid metabolism (Chen, 2021). When circulating blood glucose levels are elevated, pancreatic $\beta$ cells secrete insulin, which binds to hepatic insulin receptor (INSR). The receptor undergoes autophosphorylation, leading to the recruitment and phosphorylation of insulin receptor substrates (IRSs) that, in turn, activate downstream genes, finally resulting in AKT phosphorylation and activation. Once fully activated, AKT is involved in many downstream pathways, through which it regulates a variety of metabolic processes, including gluconeogenesis, glycolysis, glycogen synthesis, and lipid synthesis (Cherrington et al., 2007; Czech, 2017; Edgerton et al., 2017; Scherer, 2019). Insulin plays two main roles in the liver, namely, inhibiting glucose production (gluconeogenesis) and activating fatty acid and triglyceride (TG) synthesis (lipogenesis). Under the insulin resistance state, insulin does not inhibit gluconeogenesis; instead, it paradoxically overactivates adipogenesis, which leads to a fatal combination of hyperglycemia and hypertriglyceridemia (Staehr et al., 2004).

Insulin resistance in the liver is often accompanied by dyslipidemia and the occurrence of NAFLD (Byrne and Targher, 2015). The increase of fatty acid level is the main cause of hepatic steatosis and insulin resistance. Glucose feeding (and increased insulin levels) increases the production of new fat and stimulates the expression, nuclear localization, and transcriptional activity of sterol regulatory element-binding transcription factor 1 (SREBP1c) and other transcription factors (Horton et al., 2002). After activation of SREBP1c, the expression of genes related to de novo lipid synthesis, such as fatty acid synthase (FAS) and acetyl-CoA carboxylase (ACC), is upregulated, thereby increasing the production of fatty acids (Horton et al., 2002; Alves-Bezerra and Cohen, 2017). Peroxisome proliferator-activated receptor gamma $(\operatorname{PPAR} \gamma)$, another nuclear hormone receptor, contributes to energy storage primarily by promoting adipogenesis and lipid synthesis and displays the highest expression levels in white adipose tissue (WAT); meanwhile, the complete transcriptional activity of PPARs requires the binding of homologous lipid ligands and heterodimerization with retinoid-X receptor ( $R X R)$, also a nuclear receptor. PPAR $\gamma$ phosphorylation may restore insulin sensitivity by enhancing PPAR $\gamma$ function. In contrast, PPAR $\gamma$ dominant-negative mutations result in hypertension and insulin resistance, suggesting that a relationship exists between PPAR $\gamma$ function and metabolic syndrome (Plutzky, 2011; Christofides et al., 2021).

Several studies have shown that EDA, a recently identified hepatokine, is mainly expressed in the liver and can be secreted into the circulatory system to participate in energy and glycolipid metabolism (Awazawa et al., 2017; Yang et al., 2019; Bayliss et al., 2021). Awazawa et al. (2017) found that the expression 
levels of EDA, corresponding to miR-676, were higher in the livers of $d b / d b$ mice than in those of control mice. Clinical studies have shown that in humans, EDA expression in the liver is positively correlated with liver fat content, visceral fat area, and NASH scores. Additionally, EDA expression was significantly decreased after surgery, and was accompanied by weight loss and improved insulin sensitivity. A case-control study (Yang et al., 2019) showed that the serum EDA-A2 concentration in patients with NAFLD was higher than that in the controls. The frequency of NAFLD increased with increasing EDA-A2 levels. ROC curve analysis also revealed that EDA-A2 levels could predict the presence of NAFLD. Significant and positive associations were found between EDA-A2 levels and $\mathrm{BMI}$, waist-to-hip ratio (WHR), fasting plasma glucose (FPG), hemoglobin Alc (HbAlc), and homeostasis model assessment of insulin resistance (HOMA-IR), a series of anthropometric parameters and some parameters of glucose metabolism and insulin function. In addition, Bayliss et al. (2021) found that liver EDA mRNA levels were higher in NASH group than in those without NAFLD; however, no difference was detected between NAFL and non-NAFLD patients. Compared with patients without NAFLD, plasma EDA concentrations were increased in both the NAFL and NASH groups and were positively correlated with the degree of steatosis. Interestingly, the authors proposed that plasma EDA was not a reliable biomarker for NAFLD and could not discriminate between NAFL and NASH. Several reasons can explain the differences between these two studies. First, Yang et al. assessed serum EDA-A2, while Bayliss et al. assessed total serum EDA. EDAA1 and EDA-A2 share a TNF homologous domain and differ by only two amino acids. Moreover, the proportion of EDAA2 in total EDA is unclear. The second main reason relates to the different criteria used for NAFLD diagnosis. Yang et al. used ultrasonography and graded NAFLD based on the Chinese Standard, while in Bayliss's study, NAFLD was determined using liver biopsy and histological assessment. In conclusion, it remains unknown whether EDA concentrations can predict the presence of NAFLD, and additional studies are required to address the above-described discrepancies.

In experiments in vivo, evaluation based on enzyme-linked immunosorbent assay (ELISA) showed that the plasma EDA concentrations were higher in $d b / d b$ mice and obese mice than in their respective control groups (Awazawa et al., 2017). EDAA2 overexpression resulted in higher glucose concentration in the glucose tolerance test and lower energy consumption relative to control (GFP-AAV-injected) mice. Immunoblot analyses showed a comparable upregulation of insulin receptor substrate 1 (IRS1) phosphorylation at Ser307 in skeletal muscle. Similarly, suppressing EDA expression decreased blood glucose concentrations in an insulin tolerance test but did not influence weight, energy expenditure, exercise ability, or food intake. Furthermore, a different study (Yang et al., 2019) showed that EDA knockdown attenuated hepatic lipogenesis in HepG2 cells. The TG content in free fatty acid (FFA) + EDA small interfering RNA (siRNA)-treated cells was significantly lower when compared with that in cells treated with FFAs alone. The effect of EDA on liver lipid metabolism might be exerted through the regulation of lipolysis- and lipogenesis-related genes such as
SREBP1c and the key fatty acid synthesis-associated enzymes FAS and ACC. The TG content of mice fed a high-fat diet (HFD) for 8 weeks was significantly increased, while the HFD-induced increase in the numbers of lipid droplets was markedly weakened in EDA-depleted mice. In addition, EDA knockdown inhibited serum aspartate transaminase (AST) and alanine transaminase (ALT) activity, but not that of alkaline phosphatase (ALP).

The mechanism involved in how obesity leads to the upregulation of EDA expression in the liver has also been investigated. Awazawa et al. (2017) found that the combined expression of PPAR $\gamma$ and RXR- $\alpha$ induced EDA promoter activity and EDA mRNA expression in Hepa1-6 cells, while the overexpression of either factor alone or the pharmacological activation of PPAR $\gamma$ failed to induce EDA expression in the liver. JNK is one of the best-characterized signal transducers in obesity and insulin resistance (Pal et al., 2016; Solinas and Becattini, 2017). In vivo administration of EDA-A2 increased JNK phosphorylation, while JNK phosphorylation levels were found to be higher in mice injected with EDA-A2-AAV than in control mice (Awazawa et al., 2017).

Collectively, these findings suggest that EDA secreted from liver tissues is associated with insulin resistance, diabetes, and NAFLD and that stimulating EDA or blocking EDA signaling may modulate hepatic steatosis and insulin resistance; however, the specific underlying mechanisms remain elusive (Figure 2). In addition, although some studies have shown that EDA-A2 has a role in metabolic diseases, other studies have been unable to distinguish whether this regulation is exerted by EDA-A1 or EDA-A2. This highlights the need to investigate the impact of EDA-A1 and EDA-A2 on metabolic disorders separately, including the detection of their concentrations in circulating blood and their expression levels in liver tissues, as well as their specific effects using loss- and gain-of-function methods.

\section{THE ROLE OF ECTODYSPLASIN A IN DIABETIC NEPHROPATHY}

Diabetic nephropathy (DN) is a common complication in patients with diabetes. It is the main cause of chronic kidney disease $(\mathrm{CKD})$ and end-stage renal disease (ESRD). DN is characterized by podocyte apoptosis, mesangial cell proliferation, matrix expansion, and glomerular and tubulointerstitial fibrosis (Wang et al., 2021). Studies have indicated that EDA may have a role in the development of $\mathrm{DN}$. The expression of EDA2R has been reported to be upregulated in diabetic kidneys (in both type 1 and type 2 diabetes) (Watanabe et al., 2013; Brennan et al., 2018). Recently, one study also showed that the mRNA and protein expression of EDA2R was increased in both type 1 (STZ injection) and type 2 (Btbr ob/ob) diabetic mice relative to controls. Consistent with the results in animals, EDA2R was also found to be highly expressed in podocytes treated with high glucose concentrations in vitro as well as in glomerular podocytes of diabetic patients. Moreover, EDA2R might provoke podocyte injury through the generation of reactive oxygen species (ROS). EDA2R overexpression was shown to inhibit the expression of anti-apoptotic molecules such as Mcl1 and $\mathrm{Bcl}-2$ while enhancing that of pro-apoptotic molecules 


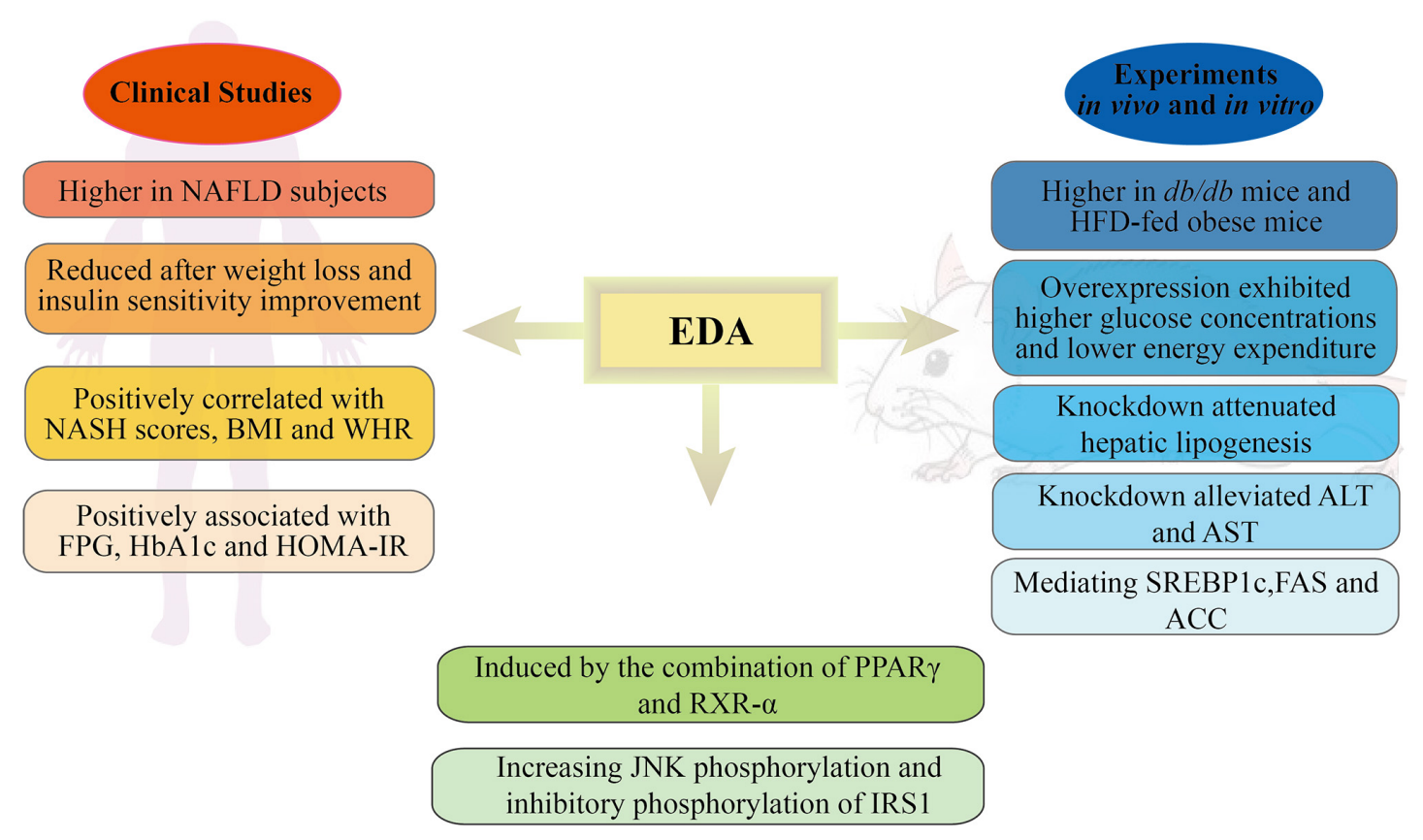

FIGURE 2 | Schematic representation of the roles and mechanisms of EDA in metabolic disorders. NAFLD, non-alcoholic fatty liver disease; NASH, non-alcoholic steatohepatitis; BMI, body mass index; WHR, waist-to-hip ratio; FPG, fasting plasma glucose; HbA1c, hemoglobin A 1c; HOMA-IR, homeostasis model assessment of insulin resistance; HFG, high-fat diet; ALT, alanine transaminase; AST, aspartate transaminase; SREBP1c, sterol regulatory element binding transcription factor 1; FAS, fatty acid synthase; ACC, acetyl-CoA carboxylase; PPAR $\gamma$, proliferator-activated receptor $\gamma$; RXR- $\alpha$, retinoid-X receptor- $\alpha$; IRS1, insulin receptor substrate 1.

such as Bax and cleaved caspase-3. EDA2R increased ROS production in podocytes, while inhibiting ROS generation could weaken EDA2R-mediated podocyte injury. In addition, EDA2Rknockdown podocytes displayed attenuated ROS production under stimulation with high glucose concentrations. The silencing of EDA2R expression partially relieved the occurrence of high glucose-induced apoptosis and dedifferentiation (Lan et al., 2020). EDA2R might exacerbate the development of DN by regulating the apoptosis and dedifferentiation of podocytes and enhancing the generation of ROS. It is not clear whether similar mechanisms mediate the involvement of EDA2R in glycolipid metabolism and DN or whether EDA2R can serve as a novel therapeutic target for the treatment of patients with DN.

\section{THE ROLE OF ECTODYSPLASIN A IN TUMORIGENESIS}

Although EDA has been proposed to play a regulatory role in cell proliferation and differentiation, this possibility remains controversial and warrants further investigation. For instance, the epidermal growth factor receptor (EGFR) gene is one of the most frequently mutated genes in lung cancer, particularly in non-small cell lung adenocarcinoma (Rosell et al., 2009). Soraas and Stebbing (2018) identified a significant and positive correlation between EDAR polymorphism and EGFR mutation frequencies, indicating that the EDAR gene might be associated with lung cancer and may have potential as a biomarker for the diagnosis of this disease. In a different study (Wang et al., 2020), the authors showed that the mRNA and protein expression of EDAR was upregulated in CRC tissues and CRC cell lines relative to their respective controls. Additionally, CRC patients with high EDAR expression have poor clinical outcomes, whereas those with low EDAR expression showed improved overall survival rates. Compared with vector controls, shRNAmediated knockdown of EDAR significantly reduced the size and number of CRC cell colonies and induced cell cycle arrest in the G1 phase. In vivo, the tumor burden of mice transplanted with shEDAR-transduced tumor cells was significantly alleviated, and the tumor volume of EDAR-deficient mice was less than $1,000 \mathrm{~mm}^{3}$. In addition, Li et al. (2020) showed that EDARADD was highly expressed in head and neck squamous cell carcinoma (HNSCC) tissues while EDARADD expression was associated with the degree of tumor differentiation and local recurrence in tongue squamous cell carcinoma (TSCC). Furthermore, EDARADD knockdown in TSCC cells affected clonogenicity, induced apoptosis, suppressed proliferation, and reduced the expression of NF- $\mathrm{Bp} 65, \mathrm{MYC}$, and Bcl-2. NF- $\mathrm{B}$ plays a broad role in cell proliferation and mediates apoptosis, especially its RelA (p65) subunit (Giridharan and Srinivasan, 2018; Zeng et al., 2019). MYC is located downstream of NF$\kappa \mathrm{B}$ and promotes cell growth and proliferation, while $\mathrm{Bcl}-2$ family proteins regulate apoptosis (Adams and Cory, 1998; Zhao et al., 2018). These results all demonstrate that both EDAR and EDARADD are involved in cancer pathogenesis; in contrast, however, Vial et al. (2019) revealed that EDAR acts as a tumor suppressor in melanoma. The authors observed a marked decrease in EDAR expression in malignant melanoma compared with that in benign nevi. Each EDAR mutation (T167I, E254K, P409L, and V416M) significantly impaired EDAR 
pro-apoptotic activity. EDAR knockout mice were reported to develop melanoma lesions over the first 400 days, a phenotype that was linked with reduced survival. Together, these findings suggest that EDA is closely linked with cancer cell proliferation, migration, differentiation, and local recurrence. Whether EDA exerts positive or negative effects is likely to be dependent on tumor type, and requires further investigation.

\section{CONCLUSION}

In summary, EDA, a hepatokine, may be associated with a variety of diseases, such as metabolic disorders, DN, and cancer, and may be a link among insulin resistance, T2DM, obesity, and NAFLD. Our review highlighted the potential roles of this hepatokine and the possibility of targeting the EDA signaling pathway in different pathophysiological processes. Importantly, elevated circulating EDA levels are closely associated with the incidence of NAFLD, rendering EDA a potential biomarker for the clinical diagnosis of this condition. However, further clinical investigation is warranted to confirm this possibility.

\section{DATA AVAILABILITY STATEMENT}

The original contributions presented in the study are included in the article/supplementary material, further inquiries can be directed to the corresponding authors.

\section{REFERENCES}

Adams, J., and Cory, S. (1998). The Bcl-2 protein family: arbiters of cell survival. Science 281, 1322-1326. doi: 10.1126/science.281.5381.1322

Alves-Bezerra, M., and Cohen, D. (2017). Triglyceride metabolism in the liver. Compr. Physiol. 8, 1-8. doi: 10.1002/cphy.c170012

Awazawa, M., Gabel, P., Tsaousidou, E., Nolte, H., Krüger, M., Schmitz, J., et al. (2017). A microRNA screen reveals that elevated hepatic ectodysplasin A expression contributes to obesity-induced insulin resistance in skeletal muscle. Nat. Med. 23, 1466-1473. doi: 10.1038/nm.4420

Bayliss, J., Ooi, G., De Nardo, W., Shah, Y., Montgomery, M., McLean, C., et al. (2021). Ectodysplasin a is increased in non-alcoholic fatty liver disease, but is not associated with type 2 diabetes. Front. Endocrinol. 12:642432. doi: 10.3389/ fendo.2021.642432

Biben, C., Wang, C., and Harvey, R. (2002). NK-2 class homeobox genes and pharyngeal/oral patterning: Nkx2-3 is required for salivary gland and tooth morphogenesis. Int. J. Dev. Biol. 46, 415-422.

Brennan, E., Mohan, M., McClelland, A., Tikellis, C., Ziemann, M., Kaspi, A., et al. (2018). Lipoxins regulate the early growth response-1 network and reverse diabetic kidney disease. J. Am. Soc. Nephrol. 29, 1437-1448. doi: 10.1681/asn. 2017101112

Byrne, C., and Targher, G. (2015). NAFLD: a multisystem disease. J. Hepatol. 62, S47-S64. doi: 10.1016/j.jhep.2014.12.012

Chen, G. (2021). The interactions of insulin and Vitamin A signaling systems for the regulation of hepatic glucose and lipid metabolism. Cells 10:2160. doi: $10.3390 /$ cells 10082160

Cherrington, A., Moore, M., Sindelar, D., and Edgerton, D. (2007). Insulin action on the liver in vivo. Biochem. Soc. Trans. 35, 1171-1174. doi: 10.1042/ bst0351171

Christofides, A., Konstantinidou, E., Jani, C., and Boussiotis, V. (2021). The role of peroxisome proliferator-activated receptors (PPAR) in immune responses. Metab. Clin. Exp. 114:154338. doi: 10.1016/j.metabol.2020.154338

\section{AUTHOR CONTRIBUTIONS}

$\mathrm{ZC}$ and GY conceived and designed the review. ZC and $\mathrm{XD}$ analyzed the data and wrote the original draft of the manuscript. DW and JJ revised the final manuscript. All authors read and approved the final manuscript.

\section{FUNDING}

This work was supported by the National Natural Science Foundation of China (81870548 and 81570721), the Social Development Project of Jiangsu Province (BE2018692), the Natural Science Foundation of Jiangsu Province, China (BK20191222 and BK20151331), the High Caliber Medical Personnel Foundation of Jiangsu Province (LGY2016053), the Six Talent Peaks Project in Jiangsu Province (2015-WSN-006), and the Fifth "169 project" Scientific Research Project of Zhenjiang City, Jiangsu Province.

\section{ACKNOWLEDGMENTS}

We thank all authors of this manuscript who have made substantial contributions to this work.

Czech, M. (2017). Insulin action and resistance in obesity and type 2 diabetes. Nat. Med. 23, 804-814. doi: 10.1038/nm.4350

de Vries, M., Faiz, A., Woldhuis, R., Postma, D., de Jong, T., Sin, D., et al. (2017). Lung tissue gene-expression signature for the ageing lung in COPD. Thorax doi: 10.1136/thoraxjnl-2017-210074 [Epub Online ahead of print].

Du, H., Zhao, Y., Yin, Z., Wang, D., and Chen, C. (2021). The role of miR-320 in glucose and lipid metabolism disorder-associated diseases. Int. J. Biol. Sci. 17, 402-416. doi: 10.7150/ijbs.53419

Edgerton, D., Kraft, G., Smith, M., Farmer, B., Williams, P., Coate, K., et al. (2017). Insulin's direct hepatic effect explains the inhibition of glucose production caused by insulin secretion. JCI insight. 2:e91863. doi: 10.1172/jci.insight.91863

Feng, J., Yang, G., Yuan, G., Gluhak-Heinrich, J., Yang, W., Wang, L., et al. (2011). Abnormalities in the enamel in bmp2-deficient mice. Cells Tissues Organs 194, 216-221. doi: 10.1159/000324644

Feng, X., Weng, C., Wei, T., Sun, J., Huang, F., Yu, P., et al. (2018). Two EDA gene mutations in chinese patients with hypohidrotic ectodermal dysplasia. J. Eur. Acad. Dermatol. Venereol. 32, e324-e326. doi: 10.1111/jdv.14874

Fliniaux, I., Mikkola, M., Lefebvre, S., and Thesleff, I. (2008). Identification of dkk4 as a target of Eda-A1/Edar pathway reveals an unexpected role of ectodysplasin as inhibitor of Wnt signalling in ectodermal placodes. Dev. Biol. 320, 60-71. doi: 10.1016/j.ydbio.2008.04.023

Giridharan, S., and Srinivasan, M. (2018). Mechanisms of NF-кB p65 and strategies for therapeutic manipulation. J. Inflamm. Res. 11, 407-419. doi: 10.2147/jir. S140188

Häärä, O., Harjunmaa, E., Lindfors, P., Huh, S., Fliniaux, I., Åberg, T., et al. (2012). Ectodysplasin regulates activator-inhibitor balance in murine tooth development through Fgf20 signaling. Development 139, 3189-3199. doi: 10. 1242/dev.079558

Han, X., Yoshizaki, K., Miyazaki, K., Arai, C., Funada, K., Yuta, T., et al. (2018). The transcription factor NKX2-3 mediates p21 expression and ectodysplasin-A signaling in the enamel knot for cusp formation in tooth development. J. Biol. Chem. 293, 14572-14584. doi: 10.1074/jbc.RA118.003373 
Headon, D., and Overbeek, P. (1999). Involvement of a novel Tnf receptor homologue in hair follicle induction. Nature genetics 22, 370-374. doi: 10.1038/ 11943

Horton, J., Goldstein, J., and Brown, M. (2002). SREBPs: activators of the complete program of cholesterol and fatty acid synthesis in the liver. J. Clin. Invest. 109, 1125-1131. doi: 10.1172/jci15593

Huh, S., Närhi, K., Lindfors, P., Häärä, O., Yang, L., Ornitz, D., et al. (2013). Fgf20 governs formation of primary and secondary dermal condensations in developing hair follicles. Genes Dev. 27, 450-458. doi: 10.1101/gad.198945.112

James, D., Stöckli, J., and Birnbaum, M. (2021). The aetiology and molecular landscape of insulin resistance. Nat. Rev. Mol. Cell Biol. 22, 751-771. doi: 10. 1038/s41580-021-00390-6

Jia, S., Kwon, H., Lan, Y., Zhou, J., Liu, H., and Jiang, R. (2016). Bmp4-Msx1 signaling and Osr2 control tooth organogenesis through antagonistic regulation of secreted Wnt antagonists. Dev. Biol. 420, 110-119. doi: 10.1016/j.ydbio.2016. 10.001

Jiang, W., Xue, P., He, Y., and Chen, Y. (2012). Molecular cloning, sequence analysis and expression of goat Eda gene. J. Northwest A\&F Univ. 40, 7-12. doi: $10.1007 /$ s11783-011-0280-z

Kere, J., Srivastava, A., Montonen, O., Zonana, J., Thomas, N., Ferguson, B., et al. (1996). X-linked anhidrotic (hypohidrotic) ectodermal dysplasia is caused by mutation in a novel transmembrane protein. Nature genetics 13, 409-416. doi: 10.1038/ng0895-409

Kumar, A., Eby, M., Sinha, S., Jasmin, A., and Chaudhary, P. (2001). The ectodermal dysplasia receptor activates the nuclear factor-kappaB, JNK, and cell death pathways and binds to ectodysplasin A. J. Biol. Chem. 276, 2668-2677. doi: $10.1074 /$ jbc.M008356200

Kwack, M., Kim, J., and Kim, M. (2019). Ectodysplasin-A2 induces apoptosis in cultured human hair follicle cells and promotes regression of hair follicles in mice. Biochem. Biophys. Res. Commun. 520, 428-433. doi: 10.1016/j.bbrc.2019. 10.031

Lan, X., Kumar, V., Jha, A., Aslam, R., Wang, H., Chen, K., et al. (2020). EDA2R mediates podocyte injury in high glucose milieu. Biochimie 174, 74-83. doi: 10.1016/j.biochi.2020.04.003

Lefebvre, S., Fliniaux, I., Schneider, P., and Mikkola, M. (2012). Identification of ectodysplasin target genes reveals the involvement of chemokines in hair development. J. Invest. Dermatol. 132, 1094-1102. doi: 10.1038/jid.20 11.453

Li, M., Bai, Y., Han, K., Li, X., and Meng, J. (2020). Knockdown of ectodysplasin-A receptor-associated adaptor protein exerts a tumor-suppressive effect in tongue squamous cell carcinoma cells. Exp. Ther. Med. 19, 3337-3347. doi: 10.3892/ etm. 2020.8578

Liu, D., Zhang, Z., He, X., Dong, Y., Zhu, Z., Bai, R., et al. (2011). FGF5 Expression and Immunolocalization in Back and Ear Skin of Young Alpaca (Lama pacos). Chin. J. Biochem. Mol. Biol. 27, 473-479. doi: 10.13865/j.cnki.cjbmb.2011. 05.009

Liu, G., Wang, X., Qin, M., Sun, L., and Zhu, J. (2019). A novel missense mutation p.S305R of EDA gene causes XLHED in a Chinese family. Arch. Oral Biol. 107:104507. doi: 10.1016/j.archoralbio.2019.104507

Liu, N., Niu, S., Cao, X., Cheng, J., Gao, S., Yu, X., et al. (2018). Let-7b regulates alpaca hair growth by downregulating ectodysplasin A. Mol. Med. Rep. 17, 4688-4694. doi: 10.3892/mmr.2018.8442

Marks, D., Penzi, L., Ibler, E., Manatis-Lornell, A., Hagigeorges, D., Yasuda, M., et al. (2019). The medical and psychosocial associations of alopecia: recognizing hair loss as more than a cosmetic concern. Am. J. Clin. Dermatol. 20, 195-200. doi: 10.1007/s40257-018-0405-2

Morinaga, H., Mohri, Y., Grachtchouk, M., Asakawa, K., Matsumura, H., Oshima, M., et al. (2021). Obesity accelerates hair thinning by stem cellcentric converging mechanisms. Nature 595, 266-271. doi: 10.1038/s41586021-03624-X

Mou, C., Jackson, B., Schneider, P., Overbeek, P., and Headon, D. (2006). Generation of the primary hair follicle pattern. Proc. Natl. Acad. Sci. U. S. A. 103, 9075-9080. doi: 10.1073/pnas.0600825103

Newton, K., French, D., Yan, M., Frantz, G., and Dixit, V. (2004). Myodegeneration in EDA-A2 transgenic mice is prevented by XEDAR deficiency. Mol. Cell. Biol. 24, 1608-1613. doi: 10.1128/mcb.24.4.1608-1613.2004

Pal, M., Febbraio, M., and Lancaster, G. (2016). The roles of c-Jun NH2-terminal kinases (JNKs) in obesity and insulin resistance. J. Physiol. 594, 267-279. doi: $10.1113 /$ jp271457
Park, J., Ko, J., and Chae, J. (2019). Novel and Private EDA mutations and clinical phenotypes of Korean patients with X-Linked hypohidrotic ectodermal dysplasia. Cytogenet. Genome Res. 158, 1-9. doi: 10.1159/000500214

Pinheiro, M., and Freire-Maia, N. (1994). Ectodermal dysplasias: a clinical classification and a causal review. Am. J. Med. Genet. 53, 153-162. doi: 10.1002/ ajmg.1320530207

Plutzky, J. (2011). The PPAR-RXR transcriptional complex in the vasculature: energy in the balance. Circ. Res. 108, 1002-1016. doi: 10.1161/circresaha.110. 226860

Rosell, R., Moran, T., Queralt, C., Porta, R., Cardenal, F., Camps, C., et al. (2009). Screening for epidermal growth factor receptor mutations in lung cancer. New Engl. J. Med. 361, 958-967. doi: 10.1056/NEJMoa0904554

Rossi, F., Bellini, G., Luongo, L., Manzo, I., Tolone, S., Tortora, C., et al. (2016). Cannabinoid Receptor 2 as Antiobesity Target: inflammation, fat storage, and browning modulation. J. Clin. Endocrinol. Metab. 101, 3469-3478. doi: 10.1210/ jc. $2015-4381$

Sadia, Foo, J., Khor, C., Jelani, M., and Ali, G. (2019). A recurrent missense mutation in the EDAR gene causes severe autosomal recessive hypohidrotic ectodermal dysplasia in two consanguineous Kashmiri families. J. Gene Med. 21:e3113. doi: 10.1002/jgm.3113

Sadier, A., Viriot, L., Pantalacci, S., and Laudet, V. (2014). The ectodysplasin pathway: from diseases to adaptations. Trends Genet. 30, 24-31. doi: 10.1016/ j.tig.2013.08.006

Scherer, P. (2019). The many secret lives of adipocytes: implications for diabetes. Diabetologia 62, 223-232. doi: 10.1007/s00125-018-4777-x

Simcocks, A., O’Keefe, L., Jenkin, K., Cornall, L., Grinfeld, E., Mathai, M., et al. (2020). The Role of Atypical Cannabinoid Ligands O-1602 and O-1918 on Skeletal Muscle Homeostasis with a Focus on Obesity. Int. J. Mol. Sci. 21:5922. doi: 10.3390/ijms21165922

Sinha, S., Zachariah, S., Quiñones, H., Shindo, M., and Chaudhary, P. (2002). Role of TRAF3 and -6 in the activation of the NF-kappa B and JNK pathways by X-linked ectodermal dysplasia receptor. J. Biol. Chem. 277, 44953-44961. doi: 10.1074/jbc.M207923200

Solinas, G., and Becattini, B. (2017). JNK at the crossroad of obesity, insulin resistance, and cell stress response. Mol. Metab. 6, 174-184. doi: 10.1016/j. molmet.2016.12.001

Soraas, L., and Stebbing, J. (2018). Geographic Variation in EGFR Mutation Frequency in Lung Adenocarcinoma May Be Explained by Interethnic Genetic Variation. J. Thorac. Oncol. 13, 454-458. doi: 10.1016/j.jtho.2017.11.128

Staehr, P., Hother-Nielsen, O., and Beck-Nielsen, H. (2004). The role of the liver in type 2 diabetes. Rev. Endocr. Metab. Disord. 5, 105-110. doi: 10.1023/B: REMD.0000021431.90494.0c

Stewart, C., and Rittweger, J. (2006). Adaptive processes in skeletal muscle: molecular regulators and genetic influences. J. Musculoskeletal Neuro. Interact. 6, 73-86.

Vial, J., Royet, A., Cassier, P., Tortereau, A., Dinvaut, S., Maillet, D., et al. (2019). The Ectodysplasin receptor EDAR acts as a tumor suppressor in melanoma by conditionally inducing cell death. Cell Death Differ. 26, 443-454. doi: 10.1038/ s41418-018-0128-1

Wang, B., Liang, Y., Chai, X., Chen, S., Ye, Z., Li, R., et al. (2020). Ectodysplasin A receptor (EDAR) promotes colorectal cancer cell proliferation via regulation of the Wnt/ $\beta$-catenin signaling pathway. Exp. Cell Res. 395:112170. doi: 10.1016/j. yexcr.2020.112170

Wang, L., Wang, H., Liu, T., and Lan, H. (2021). TGF-Beta as a Master Regulator of Diabetic Nephropathy. Int. J. Mol. Sci. 22:7881. doi: 10.3390/ijms22157881

Watanabe, T., Tomioka, N., Doshi, M., Watanabe, S., Tsuchiya, M., and Hosoyamada, M. (2013). Macrophage migration inhibitory factor is a possible candidate for the induction of microalbuminuria in diabetic $\mathrm{db} / \mathrm{db}$ mice. Biol. Pharma. Bull. 36, 741-747. doi: 10.1248/bpb.b12-00741

Wohlfart, S., Söder, S., Smahi, A., and Schneider, H. (2016). A novel missense mutation in the gene EDARADD associated with an unusual phenotype of hypohidrotic ectodermal dysplasia. Am. J. Med. Genet. Part A. 170A, 249-253. doi: 10.1002/ajmg.a.37412

Yan, M., Wang, L., Hymowitz, S., Schilbach, S., Lee, J., Goddard, A., et al. (2000). Two-amino acid molecular switch in an epithelial morphogen that regulates binding to two distinct receptors. Science 290, 523-527. doi: 10.1126/science. 290.5491 .523

Yang, J., Huang, T., Petralia, F., Long, Q., Zhang, B., Argmann, C., et al. (2015). Synchronized age-related gene expression changes across multiple tissues in 
human and the link to complex diseases. Sci. Rep. 5:15145. doi: 10.1038/ srep 15145

Yang, J., Zhou, W., Zhu, J., Wu, Y., Xu, L., Wang, Y., et al. (2019). Circulating ectodysplasin A is a potential biomarker for nonalcoholic fatty liver disease. Clin. Chim. Acta 499, 134-141. doi: 10.1016/j.cca.2019.09.009

Zeng, S., Zhao, X., Xu, L., Yang, D., Chen, L., and Xu, M. (2019). Apocynum venetumApoptosis induction effect of polyphenol on human U87 glioma cells via NF-кB pathway. Fut. Oncol. 15, 3723-3738. doi: 10.2217/fon-2019-0381

Zhang, Y., Tomann, P., Andl, T., Gallant, N., Huelsken, J., Jerchow, B., et al. (2009). Reciprocal requirements for EDA/EDAR/NF-kappaB and Wnt/betacatenin signaling pathways in hair follicle induction. Dev. Cell 17, 49-61. doi: 10.1016/j.devcel.2009.05.011

Zhao, M., Zhang, Y., Li, J., Li, X., Cheng, N., Wang, Q., et al. (2018). BIMHistone deacetylation, as opposed to promoter methylation, results in epigenetic silencing and resistance to EGFR TKI in NSCLC. Oncol. Lett. 15, 1089-1096. doi: $10.3892 / \mathrm{ol} .2017 .7411$

Zouvelou, V., Luder, H., Mitsiadis, T., and Graf, D. (2009). Deletion of BMP7 affects the development of bones, teeth, and other ectodermal appendages of the orofacial complex. J. Exp. Zool. B. Mol. Dev. Evol. 312B, 361-374. doi: 10.1002/jez.b.21262
Zurlo, F., Larson, K., Bogardus, C., and Ravussin, E. (1990). Skeletal muscle metabolism is a major determinant of resting energy expenditure. J. Clin. Invest. 86, 1423-1427. doi: 10.1172/jci114857

Conflict of Interest: The authors declare that the research was conducted in the absence of any commercial or financial relationships that could be construed as a potential conflict of interest.

Publisher's Note: All claims expressed in this article are solely those of the authors and do not necessarily represent those of their affiliated organizations, or those of the publisher, the editors and the reviewers. Any product that may be evaluated in this article, or claim that may be made by its manufacturer, is not guaranteed or endorsed by the publisher.

Copyright (C) 2021 Cai, Deng, Jia, Wang and Yuan. This is an open-access article distributed under the terms of the Creative Commons Attribution License (CC BY). The use, distribution or reproduction in other forums is permitted, provided the original author(s) and the copyright owner(s) are credited and that the original publication in this journal is cited, in accordance with accepted academic practice. No use, distribution or reproduction is permitted which does not comply with these terms. 